\title{
Sistemas Colaborativos e Educação em Computação: Uma Revisão Sistemática Sobre Comunidades de Prática e Gestão do Conhecimento no Ensino Superior
}

\author{
Lais Ribeiroํㅏ, Patrícia Scherer Bassani1 \\ ${ }^{1}$ IUniversidade Feevale \\ ERS-239, 2755 - Novo Hamburgo - RS - Brasil \\ riibeirolais@gmail.com, patriciab@feevale.br
}

\begin{abstract}
This research allies studies in the area of Collaborative Systems and Computer Education and aims to identify researches which articulate the concept of community of practice (CoPs) as spaces for knowledge management in higher education, to guide the development of a proposal for using CoPs in the context of formal education. The research, based on a qualitative and exploratory approach, involved a systematic literature review in the Web of Science (Wos) database. Results show that studies focus on the following themes: infrastructure; prototypes of communities; member's behavior; forms of content generation; and knowledge extraction and sharing.

Resumo. Este trabalho alia estudos na área de Sistemas Colaborativos e Educação em Computação e busca sistematizar pesquisas já desenvolvidas que articulam o conceito de comunidades de prática (CoPs) enquanto espaços de gestão de conhecimento no ensino superior, para subsidiar o desenvolvimento de uma proposta de uso de CoPs no contexto de ensino formal. A pesquisa, de abordagem qualitativa e exploratória, envolveu uma revisão sistemática de literatura na base de dados Web of Science (WoS). Resultados apontam que os estudos focam nos seguintes temas: infraestrutura das comunidades; protótipos de comunidades; comportamento dos membros; formas de geração de conteúdo; e extração e compartilhamento do conhecimento.
\end{abstract}

\section{Introdução}

Atualmente o conhecimento é considerado a principal fonte de domínio competitivo de uma organização. Cavusgil (2003) e Von Krogh (2000) afirmam que a aprendizagem organizacional é um processo de criação, de retenção e de transferência de conhecimento, que facilita a aprendizagem individual, bem como a aprendizagem em grupo ou em equipe. A gestão do conhecimento está geralmente relacionada com atividades como aprendizagem e inovação, benchmarking e melhores práticas, estratégia, cultura e medição de desempenho, como também com a geração, captura, armazenamento e compartilhamento de conhecimento.

As redes sociais se caracterizam como ambientes informais de aprendizagem no contexto da aprendizagem ao longo da vida (KOPER; SLOEP, 2002), uma vez que possibilitam a constituição de comunidades virtuais em torno de assuntos de interesse comum. As comunidades virtuais consistem em grupos de pessoas com preferências compartilhadas, que aprendem juntas por meio de interação e cooperação recíproca. 
Quando essas comunidades focam em aspectos educacionais, chamamos de comunidades virtuais de aprendizagem (CVA). Por outro lado, quando focam em aspectos profissionais são chamadas de comunidades de prática $(\mathrm{CoP})$.

O presente estudo busca ampliar o escopo de pesquisas na área de Educação em Computação tendo como foco o contexto das comunidades de prática. Assim, este trabalho busca sistematizar pesquisas já desenvolvidas que articulam os conceitos de CoP enquanto espaços de gestão de conhecimento. Wenger (2011) define comunidade de prática como um grupo de pessoas que compartilham uma paixão, uma preocupação ou um conjunto de problemas relativos a um determinado tópico e que interagem com a intenção de aprofundar seus conhecimentos.

Este estudo é um recorte de uma pesquisa em andamento, que busca propor um modelo de integração das CoP no contexto de ensino formal.

O artigo está assim organizado: na seção 2 apresentamos o delineamento metodológico do estudo; na seção 3, os resultados, e na seção 4, a discussão. Por fim, as considerações finais, seguidas das referências deste estudo.

\section{Metodologia}

A pesquisa foi inspirada na metodologia de revisão sistemática de literatura proposta por Kitchenham e Charters (2007). O processo de pesquisa iniciou com a definição da questão de pesquisa (RQ - research question): Quais os conceitos abordados nos estudos sobre comunidades virtuais de aprendizagem e comunidades de prática, que buscam aproximação com a gestão do conhecimento na área acadêmica?

A coleta de dados foi realizada na base de dados Web of Science (WoS), portal de periódicos disponibilizado pela CAPES. Foram selecionados apenas artigos publicados nos últimos 5 anos (2016-2020).

As palavras-chave definidas foram Virtual Learning Communities, Knowledge management, Programming Teaching, utilizadas em conjunto e separadamente, bem como fazendo associações (AND), para refinar a consulta e encontrar o maior número de artigos relevantes para o estudo. Com base nisto, gerou-se a string de busca apresentada abaixo. Esta string é aplicada no motor de buscas definido para esta revisão.

\section{(("Virtual Learning Communities") OR ("Virtual Community of Practice") OR ("Knowledge management") AND ("Programming Teaching"))}

Esta primeira etapa resultou em 67 artigos que foram extraídos da base em formato BibTex e exportados para o ambiente on-line Rayyan QCRI (https://rayyan.qcri.org/).

A seguir, fez-se a etapa de classificação, onde os artigos foram analisados à luz dos critérios de inclusão: a) ser um artigo científico publicado; b) o artigo deve apresentar uma forma de validação ou conclusão; c) a publicação deve estar disponível na íntegra na internet ou disponível através de convênios das instituições de ensino; d) o artigo deve abordar um estudo sobre comunidades virtuais de aprendizagem ou de comunidades de prática; e) o artigo deve citar sobre comportamentos ou falar sobre a utilização dentro de universidades; f) o artigo deve trazer dados concretos sobre a utilização das comunidades. 
Nesta fase foi realizada a leitura do título, palavras-chave e resumo dos 67 artigos. Ao fim desta etapa restaram 7 artigos para análise. Dentre os 60 artigos excluídos, 51 artigos foram descartados por não estarem de acordo com o assunto. Entre os descartados estavam artigos que não apresentavam um modelo de comunidades ou não tratavam sobre o assunto no contexto da área de tecnologia da informação, 2 por duplicação, e 7 pela inviabilidade de download. A próxima etapa envolveu a leitura integral dos 7 artigos selecionados. Os resultados estão organizados no Quadro 1.

Quadro 1. Organização dos trabalhos relacionados

\begin{tabular}{|c|c|c|c|}
\hline Ano & Título & Autores & Abordagem \\
\hline 2016 & $\begin{array}{l}\text { Virtual Learning Communities: } \\
\text { review of a decade of Spanish- } \\
\text { Portuguese scientific production }\end{array}$ & $\begin{array}{l}\text { Raposo-Rivas, M; Escola, J; } \\
\text { Raposo-Rivas, Manuela; } \\
\text { Escola, Joaquim; }\end{array}$ & $\begin{array}{l}\text { Pesquisa sobre } \\
\text { crescimento da } \\
\text { produção } \\
\text { científica sobre } \\
\text { CVA }\end{array}$ \\
\hline 2016 & $\begin{array}{l}\text { Participation Research of } \\
\text { Virtual Learning Community }\end{array}$ & $\begin{array}{l}\text { Zou, JM; Zhang, JP; Zou, Ju- } \\
\text { mei; Zhang, Jian-ping; }\end{array}$ & $\begin{array}{l}\text { Perfil de usuários } \\
\text { e interações nas } \\
\text { CVA }\end{array}$ \\
\hline 2017 & $\begin{array}{l}\text { Strengthening Competencies for } \\
\text { Building Software, Through a } \\
\text { Community of Practice }\end{array}$ & $\begin{array}{l}\text { Ordonez, CC; Ordonez, H; } \\
\text { Ordonez, A; Cobos, C; } \\
\text { Hernandez, G; Ordonez, } \\
\text { Cristian Camilo; Ordonez, } \\
\text { Hugo; Ordonez, Armando; } \\
\text { Cobos, Carlos; Hernandez, } \\
\text { Giovanni; }\end{array}$ & $\begin{array}{l}\text { Estratégia de } \\
\text { VCoPs em grupos } \\
\text { de } \\
\text { desenvolvimento }\end{array}$ \\
\hline 2019 & $\begin{array}{l}\text { g9toengineering: A Virtual } \\
\text { Community of Practice in } \\
\text { Knowledge Creation }\end{array}$ & Habash, R; Habash, Riadh; & $\begin{array}{l}\text { Criação de } \\
\text { conhecimento em } \\
\text { CoPs }\end{array}$ \\
\hline 2019 & $\begin{array}{l}\text { Understanding the Sense of } \\
\text { Community and Continuance } \\
\text { Intention in Virtual } \\
\text { Communities: The Role of } \\
\text { Commitment and Type of } \\
\text { Community }\end{array}$ & $\begin{array}{l}\text { Gonzalez-Anta, B; Orengo, V; } \\
\text { Zornoza, A; Penarroja, V; } \\
\text { Martinez-Tur, V; Gonzalez- } \\
\text { Anta, Baltasar; Orengo, } \\
\text { Virginia; Zornoza, Ana; } \\
\text { Penarroja, Vicente; Martinez- } \\
\text { Tur, Vicente; }\end{array}$ & $\begin{array}{l}\text { Perfil de Interação } \\
\text { dos Usuários }\end{array}$ \\
\hline 2019 & $\begin{array}{l}\text { Virtual Learning Communities: } \\
\text { Reflecting on my Teaching } \\
\text { Practicum }\end{array}$ & $\begin{array}{l}\text { Gamboa, AM; Herrera, C; } \\
\text { Maria Gamboa, Angela; } \\
\text { Herrera, Catalina GP Pixel; }\end{array}$ & Proposta de CVA \\
\hline 2019 & $\begin{array}{l}\text { Knowledge Management and } \\
\text { Reuse in Virtual Learning } \\
\text { Communities }\end{array}$ & $\begin{array}{l}\text { Houda, S; Naila, A; Samir, B; } \\
\text { Houda, Sekkal; Naila, Amrous; } \\
\text { Samir, Bennani; }\end{array}$ & $\begin{array}{l}\text { Perfil dos Usuários } \\
\text { das CVA }\end{array}$ \\
\hline
\end{tabular}




\section{Resultados}

Raposo-Rivas e Escola (2016), apresentam uma revisão sistemática que busca entender a produção científica sobre comunidades virtuais de aprendizagem entre os anos de 2005 a 2015. A pesquisa encontrou 73 documentos, em português e espanhol, em 5 palavraschaves diferentes ("Comunidade Virtual de Aprendizagem", "Comunidade Virtual", "Comunidade de Aprendizagem", "interação" ou "colaboração"). Mais de um terço dos trabalhos são contribuições que reúnem experiências e casos práticos neste nível, mostrando a perspectiva de professores e alunos. Segundo os autores, os suportes tecnológicos das CoPs avançam de plataformas como o Moodle, para as redes sociais e web 2.0 no final do período analisado, sendo essencial a existência de infraestrutura tecnológica adequadas para essas comunidades.

Zou e Zhang (2016) identificaram quatro grupos que, com base no grau de participação e valores dos membros da comunidade e seus comportamentos, também podem ser classificados em quatro tipos: trocável, informativo, sociável e egoísta. Os primeiros são os indivíduos que trocam as orientações, informações e o convívio social. Os segundos concentram-se na busca de informações e raramente entram em contato com os outros membros da comunidade. Os Sociáveis são considerados muito importantes para a comunicação e o contato entre os membros e suas trocas de informações. Para os Egoístas, não há destaque para a troca de informações e o convívio social, apenas para a necessidade do ego de ingressar nas comunidades virtuais. Quando um membro busca uma comunidade virtual, ele deseja obter alguma experiência ou está interessado em alguma atividade da comunidade. Durante o processo, o membro mudava seu papel e comportamento.

Houda, Naila e Samir (2019) tentaram entender o perfil de quem utiliza as comunidades de aprendizagem já existentes e consagradas. Também determina que o conteúdo seja gerado pelos usuários da comunidade, em forma de textos, imagens ou vídeos. No contexto do estudo, a principal forma de geração de conteúdo é em texto em diferentes tipos de conteúdo (dúvidas, tarefas de resolução de problemas, dicas e conselhos), o que é considerado uma rica fonte de conhecimento, e depois é reutilizada pelos outros usuários. O objetivo do trabalho é a implementação de um processo que contribua com a gestão do conhecimento e reutilização do mesmo nessas comunidades on-line, de forma a extrair conhecimento textual de conteúdo gerado por usuários na comunidade on-line, para capitaliza-lo e armazená-lo em uma base de conhecimento bem organizada e potencializar sua reutilização por meio de uma disseminação ativa desse conhecimento.

Por fim, analisamos outros dois estudos que propuseram uma comunidade virtual. Gamboa e Herrera (2019) propuseram uma comunidade virtual de aprendizagem (CVA) como forma alternativa de ajudar os formandos a fortalecer seus conceitos, refletir e ter consciência de sua prática docente por meio da interação com os demais. A CVA foi criada para promover a reflexão dos professores em formação sobre seus próprios conhecimentos. Sua plataforma foi desenvolvida baseada em nuvem, de forma que os alunos possam ter acesso facilmente a ela. A intenção da CVA é promover o trabalho de forma colaborativa, de modo que passou a ser a ferramenta para que eles refletissem de forma consciente sobre as ações que desenvolveram em suas aulas, como estão ligados às teorias que estudaram. Já González-Anta et al (2019) tiveram como objetivo identificar a interação dos usuários em uma comunidade virtual de aprendizagem. A amostra foi 
composta por 299 participantes de três comunidades virtuais diferentes, sendo 61,5\% dos participantes menores de 30 anos. A amostra foi por meio de método de amostragem de conveniência com base na participação das comunidades virtuais, de diferentes temas e áreas. O principal objetivo da pesquisa era avaliar até que ponto o usuário se sente membro, se identifica com a comunidade e tem a percepção de pertencimento. O estudo mostra uma perspectiva valiosa para um entendimento aprofundado do comportamento dos membros nas comunidades. Como conclusão, o estudo indica que sustentar e desenvolver uma comunidade virtual requer fomentar um senso de comunidade e compromisso que envolva os participantes.

O estudo desenvolvido por Ordoñez et al (2017) buscou uma estratégia para compreender e formular uma estratégia para a utilização das $\mathrm{CoP}$ em grupos de desenvolvimento. $\mathrm{O}$ processo começou visitando as universidades de computação no sul da Colômbia, explicando e motivando os alunos a utilizarem as CoP. Alunos do oitavo ao décimo semestre das instituições foram convidados a participar da experiência, incluindo as disciplinas relacionadas ao desenvolvimento de software. Os participantes foram avaliados em graus de competências de um profissional da área (Resolução de Problemas, Técnicas e Ferramentas de Programação, entre outras) e o nível do desempenho foi estabelecido com graus de muito alto, alto, baixo, etc. A pesquisa teve resultados satisfatórios. Considerando o impacto, $97,1 \%$ dos integrantes afirmaram que aumentaram seus conhecimentos. Aqueles que afirmaram não terem tido resultados destacaram que foi devido a falta de tempo ou interesse de participar ativamente da CoP. Esses resultados mostram o impacto que uma CoP tem em uma instituições de Ensino Superior, auxiliando no aumento do nível das competências de seus alunos.

Habash (2019) definiu que a prática é a forma onde os alunos empregam o que aprendem e as comunidades de prática como o local onde eles podem desempenhar o papel de consumidores e produtores de conhecimento. Ele ainda cita que essas comunidades são novas formas de aprendizagem social, são comunidades de aprendizagem, conhecimento e prática colaborativa, e essas comunidades estão entre as principais práticas de criação de conhecimento em organizações e enfatiza os aspectos sociais incluídos nos processos de construção, compartilhamento e aplicação do conhecimento. Em suas pesquisas, o autor ainda define que na educação de hoje em dia os alunos não devem aprender apenas sentados nas aulas ouvindo o professor, memorizando informações e preparando respostas. Eles devem pensar sobre o que estão aprendendo, escrever sobre isso, refletir sobre experiências anteriores e aplicar o que aprenderam.

A Figura 1 apresenta a sistematização dos resultados. 
Figura 1. Sistematização dos resultados

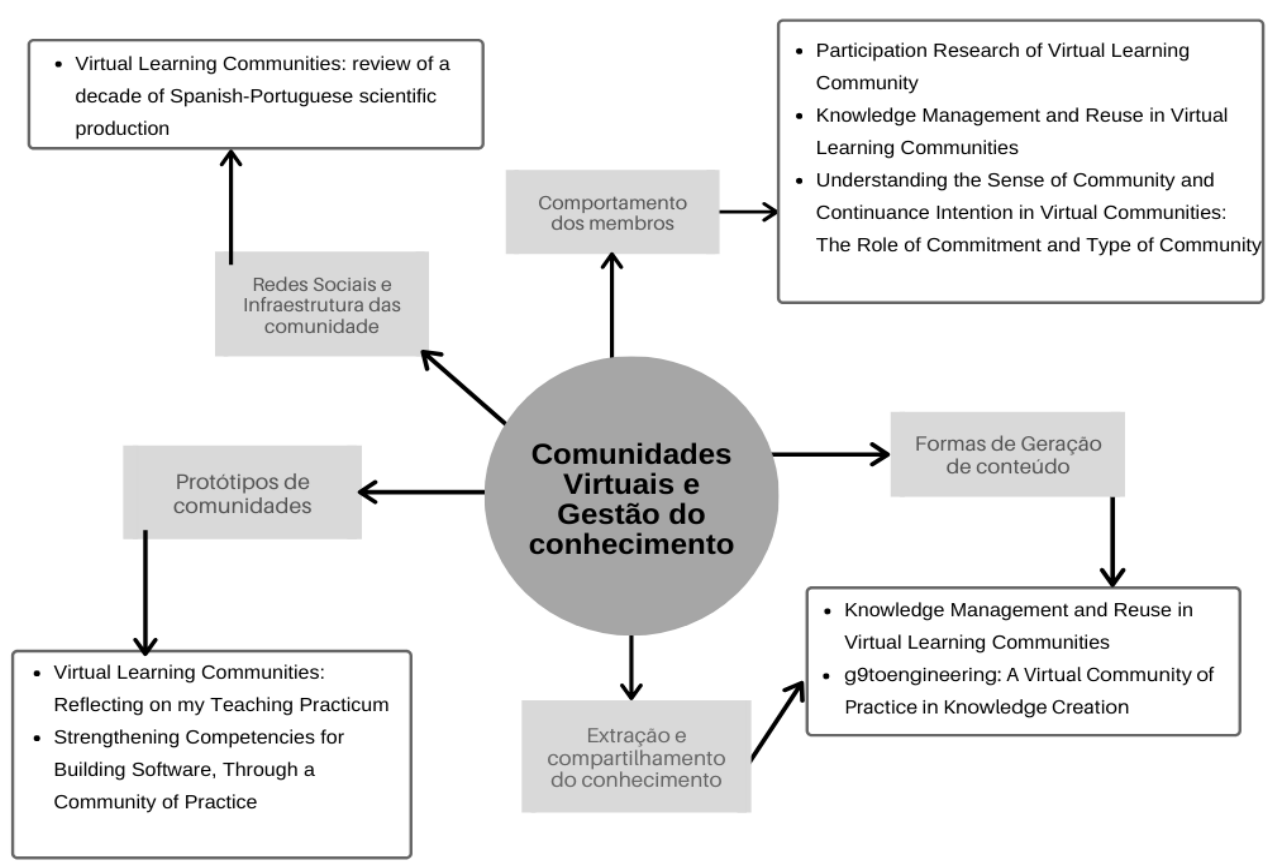

Fonte: Elaborado pelas autoras

\section{Discussão}

De acordo com a teoria da Criação do Conhecimento (NONAKA; TAKEUCHI, 1997), existem duas dimensões a serem consideradas, epistemológica e ontológica. A dimensão da criação de conhecimento epistemológico consiste em conhecimento tácito e explícito, enquanto a criação de conhecimento ontológico se preocupa com os níveis de conhecimento e criação de entidades nos níveis individuais, grupos e organizações. $\mathrm{O}$ autor também cita que o conhecimento tácito e explícito, convertidos em conhecimento organizacional por meio da socialização, externalização e combinados com modelo de internalização de criação de conhecimento, é visto como um motor de inovação contínua. Habash (2019) define que a razão pela qual as CoP são eficazes na criação de conteúdo é o fato de que a vantagem competitiva de uma organização é o conhecimento tácito intangível de seu pessoal, ou seja, não apenas novos conhecimentos são criados por meio do compartilhamento de conhecimento em $\mathrm{CoP}$, mas habilidades também são indiretamente produzidas e divulgadas durante as atividades desenvolvidas na rede. As CoP também desempenham um papel crítico na promoção da inovação e podem ser uma ferramenta muito poderosa para criar benefícios competitivos sustentáveis, especialmente na construção de equipes.

A geração de conhecimento em CoP é condicionada por vários fatores que incluem os individuais, coletivos, organizacionais ou tecnológicos. Os indivíduos envolvidos na criação do conhecimento são motivados por meio do sentimento de realização ao compartilhar conhecimento com outras pessoas. O coletivo está relacionado à comunidade (coletivo), ao contexto em que os membros sentem a obrigação moral de retribuir o que "ganharam". 
Portanto, a partir da pesquisa conduzida destacamos indicadores que podem orientar uma proposta de modelo para a inserção de uma proposta de CoP no contexto da Educação em Computação:

- traçar perfis de usuários, a fim de entender como cada papel será conduzido dentro da comunidade e de que forma cada usuário irá contribuir para inserção de conhecimento;

- ter um modelo que condiz com as redes já existentes, incentivando a troca de conhecimento e informação, e a interação entre os usuários;

- a comunidade precisa gerar o sentimento de pertencimento ao usuário, o mesmo precisa saber que seu conhecimento e sua contribuição são importantes para seu desenvolvimento e dos demais membros.

\section{Considerações finais}

Este estudo partiu da seguinte questão: Quais os conceitos abordados nos estudos sobre comunidades virtuais de aprendizagem e comunidades de prática que buscam aproximação com a gestão do conhecimento na área acadêmica?

A partir da revisão sistemática realizada identificamos que os estudos focam nos seguintes temas: infraestrutura das comunidades; protótipos de comunidades; comportamento dos membros; formas de geração de conteúdo; e extração e compartilhamento do conhecimento.

Pode-se constatar que existem esforços por parte dos profissionais na melhoria da gestão do conhecimento na área acadêmica, além de mostrar que este é um tema em ascensão na comunidade acadêmica, uma vez que dos sete estudos analisados, quatro são de 2019.

No conjunto de trabalhos analisados não foram identificados trabalhos com foco em modelos direcionados ao ensino de programação, área principal que será abordada nas próximas etapas da pesquisa. Entretanto, destacamos o volume de trabalhos analisados como uma limitação da pesquisa, uma vez que a ampliação da consulta a outras bases de dados podem desvelar um cenário diferente.

Nesse caso, entendemos que aprofundar estudos na área de comunidades de aprendizagem e seus usos no contexto de ensino superior formal encontra espaço fértil para debate.

\section{Referências}

CAVUSGIL, S. Tamer; CALANTONE, Roger J.; ZHAO, Yushan. Tacit knowledge transfer and firm innovation capability. The Journal of Business and Industrial Marketing, v. 18, n. 1, p. 6-21, 2003.

DE ANDRADE, Ilza Almeida et al. Inteligência coletiva e ferramentas web 2.0: a busca da gestão da informação e do conhecimento em organizações. Perspectivas em Gestão \& Conhecimento, v. 1, n. 1, p. 27-43, 2011.

GAMBOA, Ángela María; HERRERA, Catalina. Virtual Learning Communities: Reflecting on my Teaching Practicum. 
GONZÁLEZ-ANTA, Baltasar et al. Understanding the Sense of Community and Continuance Intention in Virtual Communities: The Role of Commitment and Type of Community. Social Science Computer Review, p. $0894439319859590,2019$.

GAMBOA, Ángela María; HERRERA, Catalina. Virtual Learning Communities: Reflecting on my Teaching Practicum.

HABASH, Riadh. g9toengineering: A Virtual Community of Practice in Knowledge Creation. In: 2019 IEEE Global Engineering Education Conference (EDUCON). IEEE, 2019. p. 1504-1511.

KOPER, Rob; SLOEP, Peter. Learning Networks: connecting people, organizations, autonomous agents and learning resources to establish the emergence of effective lifelong learning. 2002.

MACHADO, Felipe Nery Rodrigues. Big Data O Futuro dos Dados e Aplicações. Editora Saraiva, 2018.

ORDOÑEZ, Cristian Camilo et al. Strengthening competencies for building software, through a community of practice. In: Colombian Conference on Computing. Springer, Cham, 2017. p. 415-426.

RAPOSO-RIVAS, Manuela et al. Comunidades Virtuales de Aprendizaje: revisión de una década de producción científica Hispano-Lusa. 2016.

SANTORO, F. M.; PIMENTEL, M. Tecnologias Computacionais para Educação. Chronos (UNIRIO), v. 1, p. 83-91, 2009.

SEKKAL, Houda; AMROUS, Naila; BENNANI, Samir. Knowledge management and reuse in virtual learning communities. International Journal of Emerging Technologies in Learning (iJET), v. 14, n. 16, p. 23-39, 2019.

VON KROGH, Georg et al. Enabling knowledge creation: How to unlock the mystery of tacit knowledge and release the power of innovation. Oxford University Press on Demand, 2000.

WENGER, Etienne. Communities of practice: A brief introduction. 2011.

ZOU, Ju-Mei; ZHANG, Jian-Ping. Participation Research of Virtual Learning Community. In: 2016 3rd International Conference on Mechatronics and Information Technology. Atlantis Press, 2016. 